\title{
Detection of indoor corridor walls using line laser and camera
}

\author{
Miki Suetsugu*, Shiyuan Yang, Seiichi Serikawa \\ Department of Electrical and Electronic Engineering, Kyushu Institute of Technology, Kitakyushu, Japan \\ *Corresponding Author: p108060m@mail.kyutech.jp
}

\begin{abstract}
This research proposes a system that can easily and widely detect indoor corridor walls with a single system using a line laser and a camera. In order to safely use an automated guided vehicle indoors, it is necessary to detect a wall and avoid a collision. Conventional automatic guided vehicles have a detection method using a plurality of sensors or a detection method using LiDAR. However, these have the problem of being easily affected by disturbance and the problem that the sensor itself is expensive. In this study, we constructed a simple system by using a line laser and a camera mounted on an automatic moving cart, and performed experiments under the conditions moving straight in parallel to the wall and toward the wall. Experimental results showed that it can be performed easily with widely detection area in one system.
\end{abstract}

Keywords: Electronic, Robot, Sensor, Image processing, Camera, Laser, Detection, Measurement.

\section{Introduction}

In warehouses and factories, humans and robots often work together and need to coexist well. Therefore, detection of corridor walls is indispensable. For detection, it is necessary to instantaneously detect the surrounding situation over a wide area.

Ultrasonic sensors, PSD sensors, cameras, and 2DLiDARs are currently used as obstacle detection systems ${ }^{(1)}$. However, they have some disadvantages. Ultrasonic sensors can only measure in one dimension. A large number of ultrasonic sensors are needed to measure a wide range when using ultrasonic sensor method ${ }^{(2)}$. In addition, an error occurs because it is easily affected by ambient noise. Errors occur in PSD sensors due to the effect of light reflected from the floor $^{(3)}$. Also, this method requires a large number of sensors to measure over a wide range, like the ultrasonic sensor. There are also various methods using cameras. For example, there are methods of detecting an obstacle from a difference between a preset background image and an input image ${ }^{(4)-(7)}$. This image processing method is troublesome because it is necessary to take in a background image in advance. There is also a method that uses a spot laser and a rotating mirror to scan the surroundings at high speed and measure the environment ${ }^{(8)}$. Since it has a mechanical drive section called a rotating mirror, its life is shorter than a sensor composed of electronic components. It is also expensive. In order to solve these problems, we have proposed a method to detect various obstacles using a line laser and a camera. With this method, the device can be constructed relatively inexpensively. In addition, an object can be detected at high speed. However, this method cannot measure the surrounding environment such as a wall.

Therefore, in this research, we propose a method for recognizing a wall that is a large frame in a room. By combining this method with previous research, autonomous robots can be run more flexibly.

\section{Wall recognition method}

\subsection{System configuration}

Fig. 1 shows an outline of the system configuration. As shown in Fig. 1, in this research system, a green line laser is mounted on the robot and illuminates the floor. The state is photographed with a camera attached to the bottom of the robot. Fig. 2 shows an example of the obtained image.

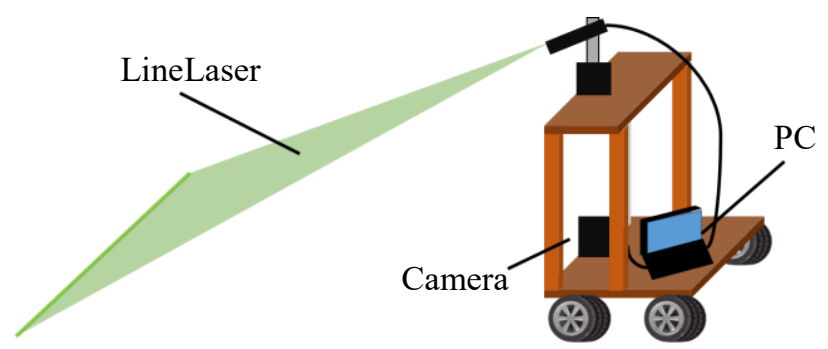

Fig. 1. Configuration of this research system 


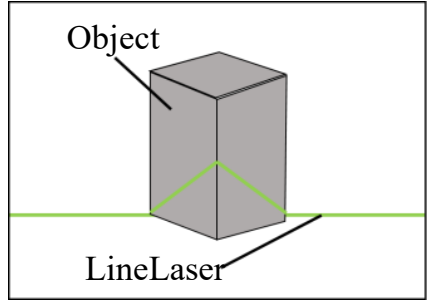

Fig. 2. Line laser irradiation

\subsection{Line laser line extraction principle}

The green line laser shown in Fig. 2 is extracted by image processing as follows. As preprocessing, an image is cut out only to a necessary area. Grayscale the cropped image. Line laser can be extracted by detecting a straight line. Fig. 3 shows the steps of the preprocessing. Step 1 is an image obtained by photographing the state of the line laser in FIG. 1 with a camera. This green line is the line laser. Since the distance for irradiating the line laser is set in advance, it is possible to cut out only the line laser portion from the focal length of the lens of the camera and the position for irradiating the line laser. The image cut out in step 2 is shown. In step 3, the image cut out only at the line laser portion is subjected to gray scale processing. The brightness value of the laser is close to 0 because the light is very strong compared to the illumination light in the room. In step 4, a pixel having a luminance value close to 0 is focused and straight line detection processing is performed.

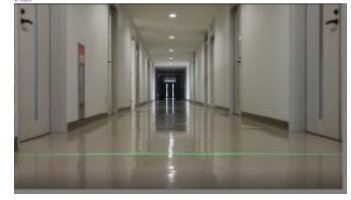

Step1. original
Step2. trimming

\section{Step3. grayscale Step4. Line detect}

Fig. 3. Line laser extraction procedure

\subsection{Principle of wall detection in indoor hallways}

In this section, the wall is detected using the line laser extracted in section 2.2. Fig. 4 shows an image obtained by photographing the state where the line laser is irradiated by the camera.

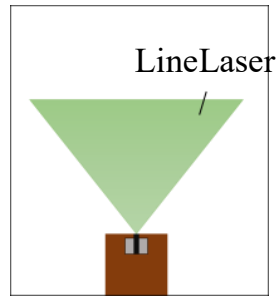

(a)

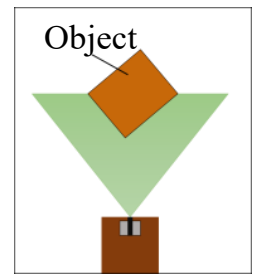

(b)

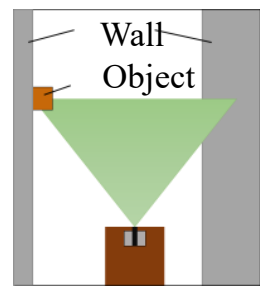

(c)

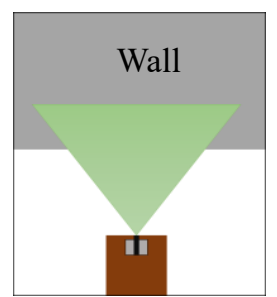

(d)

Fig.4. State image of the irradiated line laser

The left side of Fig. 4 shows all situations where the line laser is irradiated. The right side of Fig. 4 shows an image captured by the camera in that situation. The image of step 2 in Fig. 3 shows four patterns in all situations on the right side of Fig4. Fig.4 (a) shows the state of the line laser when there is no target in front of the robot. Green indicates the line laser. If there is no object, the line laser is straight in the image taken by the camera. In the image on the right side of Fig.4 (b), the lines are interrupted. In this case, it is detected that an obstacle exists in front of the robot. In the image on the right side of Fig.4 (c), the end of the line laser is interrupted. In this case, it can be determined that there is an obstacle or a wall in front of the robot. The image on the right side of Fig.4 (d) has no line laser. As described above, when the line laser cannot be detected from the cutting range set in advance, it can be determined that there is a wall or a large-sized obstacle in front of the robot. 


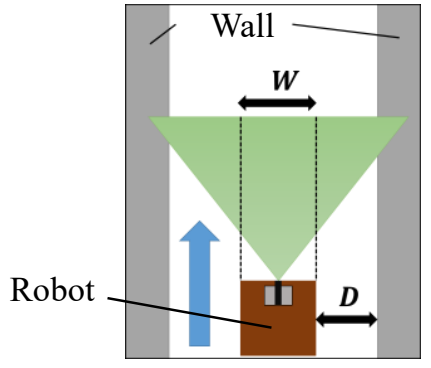

(a)

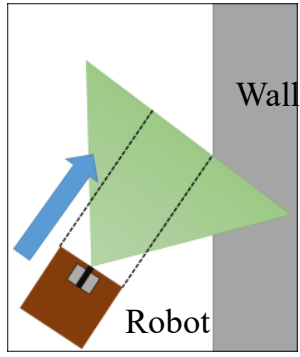

(b)
Fig.5. Driving direction of bogie

Fig. 5 shows the state of the line laser with respect to the traveling direction of the bogie. The blue arrow indicates the direction of travel of the robot. The width $\mathrm{W}$ of the black broken line in the figure indicates the width of the robot. D in Fig. 5 indicates the distance between the wall and the robot. Fig.5 (a) shows a case where the cart goes straight in parallel with the wall, and Fig.5 (b) shows a case where the cart goes toward the wall. If the line laser is not straight within the range $\mathrm{W}$ with respect to the traveling direction of the carriage, it is determined that there is a risk of collision.

\section{Experiment and discussion}

\subsection{Overview of the experimental system}

Fig. 6 shows a schematic diagram of the system proposed in this study. These are composed of a line laser in which a semiconductor laser is linearly polarized using a cylindrical rod lens, a camera, and a personal computer. As shown in Fig. 6 , the camera is installed at a low position on the cart, and the line laser is installed at a high position. The width of the robot is $40[\mathrm{~cm}]$. The camera shoots the line laser on the floor and inputs it to a computer. In this study, the camera is EOS M10 manufactured by Canon and the semiconductor laser is 520 [mm] wavelength, and is MLXL series of Kiko Giken.

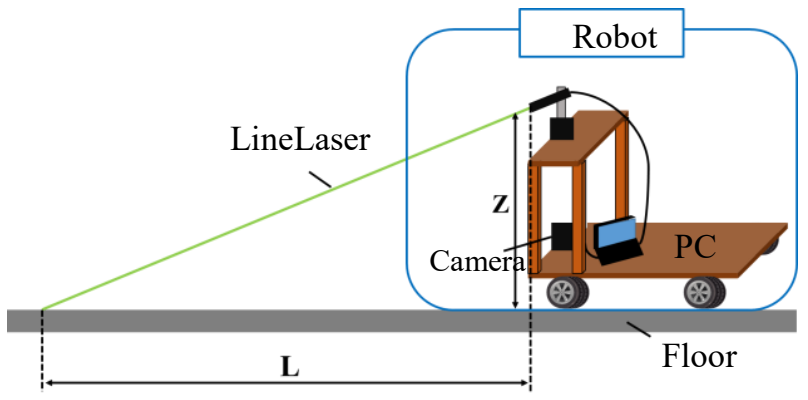

Fig.6. System outline diagram

\subsection{Verification when going straight in parallel with the wall}

In this experiment, the distance $\mathrm{L}$ between the irradiation destination of the line laser and the robot was set to $1.5[\mathrm{~m}]$. When going straight in parallel with the wall, verify that it is judged that there is a risk of colliding with the wall of the indoor corridor. If the line laser is not straight within the range of $\mathrm{W}$ in Fig. 5 with respect to the traveling direction of the bogie, it is determined that there is a risk of collision. As shown in Fig.2, when irradiating an obstacle or a floor with a line laser, the line laser is not linear. Lines break or shift. In Fig.4(d), if there is a wall in front of the robot, the line laser cannot be confirmed in the process of Fig.3 (step 2). The experiment was performed under three conditions of distance $\mathrm{D}$ to the wall being $20[\mathrm{~cm}], 50[\mathrm{~cm}]$, and $100[\mathrm{~cm}]$. Table 1 shows the experimental results.

Table 1. Verification results when traveling straight parallel to the wall

\begin{tabular}{|c|c|}
\hline $\begin{array}{l}\text { Distance between wall and robot } \\
{[\mathrm{cm}]}\end{array}$ & $\begin{array}{l}\text { Risk of collision and } \\
\text { judgment }\end{array}$ \\
\hline 20 & $\times$ \\
\hline 50 & $\times$ \\
\hline 100 & $\times$ \\
\hline
\end{tabular}

From the results in Table 1, it was not determined that there was a risk of collision under all conditions. Therefore, it is considered that there is no possibility that this research system recognizes that there is a risk of accidentally colliding.

\subsection{Verification when proceeding toward a wall}

Next, the case of going toward the wall was verified. The basic conditions are the same as in the previous section. The distance $\mathrm{L}$ between the irradiation destination of the line laser and the robot was set to $1.5[\mathrm{~m}]$. When proceeding toward a wall, verify that it is judged that there is a risk of collision with the wall of the indoor corridor. It is determined that there is a risk of collision when the line laser deviates within the range of $\mathrm{W}$ in Fig.5 with respect to the traveling direction of the bogie. When it is determined that there is a risk of collision, verification is performed to determine whether the collision was determined before the collision. The starting position starts from the direction parallel to the wall as in the previous section. The experiment was performed under three conditions of distance $\mathrm{D}$ to the wall being $20[\mathrm{~cm}], 50[\mathrm{~cm}]$, and $100[\mathrm{~cm}]$. After starting, we experimented toward the wall. Table 2 shows the experimental results. 
Table 2. Verification results when proceeding toward the wall

\begin{tabular}{|c|c|c|}
\hline $\begin{array}{l}\text { Distance between wall } \\
\text { and cart (starting point) } \\
\text { [cm] }\end{array}$ & $\begin{array}{l}\text { Risk of collision } \\
\text { and judgment }\end{array}$ & $\begin{array}{l}\text { Judge before } \\
\text { collision }\end{array}$ \\
\hline 20 & $\bigcirc$ & $\bigcirc$ \\
\hline 50 & $\bigcirc$ & $\bigcirc$ \\
\hline 100 & $\bigcirc$ & $\bigcirc$ \\
\hline
\end{tabular}

From the results in Table 2, it was determined that there was a risk of collision under all conditions. In addition, it was possible to judge before collision under all conditions. Therefore, this research system can recognize that there is a danger of collision before collision, and it is considered to be highly useful as a system for recognizing walls in indoor mobile robots.

\section{Conclusions}

Currently, the demand for indoor mobile robots is increasing. Therefore, a system for detecting an indoor wall is indispensable, and a highly accurate system is required. In the conventional method, a plurality of sensors are incorporated and expensive sensors are used. However, this research system was composed of a line laser and a camera, and a low-cost and high-accuracy system was realized.

The method proposed in this study depends on the irradiation part of the line laser. Therefore, a portion not irradiated with the line laser cannot be detected. Improving this point is an issue for the future.

\section{References}

(1) Han Wang, Zhuo Wei, Sisong Wang, Chek Seng Ow, Kah Tong Ho and Benjamin Feng: "A vision-based obstacle detection system for Unmanned Surface Vehicle”, IEEE, 5, pp.364-369, 2011

(2) Hidenori Ishihara, Toshio Fukuda, Kazuhiro Kosuge and F.Arai : "An approach to autonomous microrobot micro line trace robot with reflex algorithm", IEEE /ASME Transactions, Vol. 1, No. 1, pp.68-79, 1996

(3) Jeong-Gi Lee, D.Kim, Jin-Young Suk: "Development of a 3D Position Determination Device using PSD sensor", IEEE, pp.1733-1736, 2010

(4) Kosei Matsumoto, Toshio Moriya and Kazuo Yano: "Obstacle avoidance system for a user guided mobile robot”, United States Patent, pp.245-264, 2011

(5) Oe, Motoko, Tomokazu Sato, Naokazu Yokoya:
"Estimating camera position and posture by using feature landmark database.”, Scandinavian Conference on Image Analysis. Springer Berlin Heidelberg, pp. 171181,2005

(6) Florin Oniga, Sergiu Nedevschi: "Processing Dense Stereo Data Using Elevation Maps: Road Surface, Traffic Isle, and Obstacle Detection”, IEEE, 59, pp.1172-1182, 2009

(7) Joydeep Biswas and Manuela Veloso: "Depth camera based indoor mobile robot localization and navigation", IEEE, pp.1967-1702, 2012

(8) Nils Gageik, Paul Benz and Sergio Montenegro: "Obstacle Detection and Collision Avoidance for a UAV With Complementary Low-Cost Sensors", IEEE, Volume3, pp.599-609, 2015 